\title{
The Cold Pressor Test as a Predictor of Prolonged Postoperative Pain, a Prospective Cohort Study
}

\author{
Cameron MacLachlan · Edward A. Shipton · J. Elisabeth Wells
}

Received: July 9, 2016 / Published online: September 20, 2016

(C) The Author(s) 2016. This article is published with open access at Springerlink.com

\begin{abstract}
Introduction: Presently, it is difficult to predict which patients are at increased risk of ongoing pain problems postoperatively. This study followed a group of patients from the week before their operation until 3 months after it, to identify potential risk variables.
\end{abstract}

Methods: Fifty-four patients undergoing moderate-major gynaecological surgery at Christchurch Women's Hospital were recruited and assessed preoperatively over an 11-week period. At this initial assessment, participants were subjected to a cold pressor test (CPT). Telephonic follow-up was conducted at 6 weeks and 3 months postoperatively, to determine pain status. Information regarding the type of

Enhanced content To view enhanced content for this article go to http://www.medengine.com/Redeem/ CE76F06029DD9B31.

C. MacLachlan · E. A. Shipton ( $\square)$

Department of Anaesthesia, University of Otago, Christchurch, New Zealand

e-mail: shiptonea@xtra.co.nz

J. E. Wells

Department of Biostatistics and Computational Biology, University of Otago, Christchurch, New Zealand operation and surgical approach was collected from hospital records.

Results: Pain threshold (time taken to report the onset of pain), as measured by the CPT, was significantly predictive of prolonged pain outcomes (area under the curve $=0.80,95 \%$ CI 0.66, 0.95). Pain tolerance (total time taken to end the CPT voluntarily) was similarly predictive but non-significant (area under the curve $=0.69,95 \%$ CI 0.47, 0.90).

Conclusion: The preoperative cold pressor test shows some promise for predicting ongoing postoperative pain. However, more research is needed to determine the clinical significance of these findings in larger samples and how they could be incorporated into clinical practice.

Keywords: Cold pressor test; Pain; Postoperative; Prolonged

\section{INTRODUCTION}

Tissue insults and the associated pain are naturally a necessary and essentially ubiquitous part of surgical intervention. For most patients, this pain dissipates within a few days or weeks postoperatively. However, for 
poorly understood reasons, a sizeable minority experience prolonged (or "chronic") pain problems [1-3]. These pain states have been shown to have a large negative impact not only on the sufferer, but on the healthcare system and economy as a whole [1-5].

Although it is recognized that medical and surgical procedures may lead to persistent postoperative pain [1-3], predicting which patients are at risk for developing chronic pain presents an ongoing challenge [6]. It is hoped that reliable and timely (preoperative) identification of at-risk individuals may allow for the development and rational use of preventative interventions.

Much of the work to date regarding the prediction of postoperative pain has focussed on predicting the intensity of acute pain (pain during the "normal" healing period) [7-10]. There is increasing interest in the prediction of prolonged pain state development as well $[1-3,6]$. Most have focussed on a wide variety of demographic, psychological, surgical, and anaesthetic variables (in a wider variety of settings and ways) [1-3, 7, 8]. While genetics and neurobiology are often mentioned as important factors, they are seldom experimentally quantified. However, several experimental or physiological sensory tests have emerged, often using temperature extremes to measure a participant's resilience to pain. Preoperative results of these tests have been shown to predict the severity of acute postoperative pain [9-12]. Preoperative pain tests may predict anywhere between $4 \%$ and $54 \%$ of the variance in subjective postoperative pain intensity, depending on the stimulation methods and the test paradigm used [13]. The predictive strengths reported for these physiological tests are much higher than any previously reported for single-factor analyses of demographic and psychological factors [13].
Perioperative assessment of an individual's capacity to withstand pain could there potentially provide clues as to their risk of developing a prolonged pain state postoperatively.

Experimental stimulation methods include: the cold pressor test, heat immersion test, brief phasic or tonic heat stimulation, cutaneous electrical stimulation, pressure algometry, punctate mechanical stimulation, and induction of an inflammatory injury [13]. Supra-threshold heat pain has been found to be the most consistent test modality [14]. The cold pressor test provides a valid and low-risk test for evaluating pain in preoperative patients and has been routinely used in pain research [7]. The test is extensively documented in the pain research and in cardiac physiology [12, 15-17].

\section{Aims}

The aims of this study included, firstly, to determine whether or not any (or all) of the measures recorded during preoperative administration of the cold pressor test could be predictive of the development of a prolonged pain state. Secondly, factors related to the surgery such as anaesthetic technique [18] and surgical risk factors such as duration of surgery, type of surgery, and injury of other major nerves [19] were recorded as possible predictors of prolonged pain, as these have been reported to predict the development of chronic pain. Demographic and psychological predictors of chronic pain have already been reported for this cohort [8]. The data obtained in this study are from the same prospective cohort that we have previously published from different predictors of chronic pain [8]. In that publication, we examined the prevalence of the transition of acute pain to pathological chronic pain at 6 weeks and 3 months postoperatively [8]. 
Common emotions experienced by patients scheduled for surgery are anxiety and fear. High preoperative anxiety, surgical fear, and depression are predictors of severe postoperative pain [20]. Less frequent factors include a low expectation of return to work, hypochondriasis and somatization, avoidance coping, psychological vulnerability, and neuroticism [20]. Participants were given psychometric questionnaires at each time-point [8]. Psychological questionnaires used included the Depression Anxiety Stress Scale-21 (DASS-21); the Brief Illness Perception Questionnaire; the Pain treatment Satisfaction Scale; and the Short-Form McGill pain questionnaire [8]. We found the Brief Illness Perception Questionnaire (BIPQ) to be predictive of developing prolonged postoperative pain. No demographic factor was found to significantly predict the development of "prolonged pain" [8].

\section{METHODS}

This prospective cohort study was undertaken using a group of patients $(n=54)$ undergoing moderate to major gynaecological surgery at Christchurch Women's Hospital, Christchurch, New Zealand. Ethical approval was obtained from the University of Otago Human Ethics Committee (Health; Reference number HE13/ 07), and from the University of Otago, Christchurch Maori Research Advisor. We have earlier described the methodology of data collection in another paper published in this journal [8].

Participants were approached at the Christchurch Women's Hospital pre-admission clinic, within a week of their scheduled surgical date. Once consented, participants were asked to rate their pain (if any) on a 0-10 numeric rating scale (NRS). Patients were classified as "in pain" if the pain was directly related to the reason for their surgery and greater than or equal to $3 / 10$ on the VRS. The cold pressor test apparatus itself consisted of an 8-L bucket, half-filled with ice, with a metal mesh over the ice to prevent ice burns. Cold water was then poured over the ice until it filled up above the mesh at a level deep enough to cover the participant's hand. In the cold pressor test, the water temperature was monitored (maintained at $4{ }^{\circ} \mathrm{C}$ ), as a $2{ }^{\circ} \mathrm{C}$ difference in temperature can affect tolerance time and pain intensity $[7,12]$. All procedures followed were in accordance with the ethical standards of the responsible committee on human experimentation (institutional and national) and with the Helsinki Declaration of 1964, as revised in 2013. Informed consent was obtained from all patients for being included in the study.

First, the participant was briefed by the observer on how to take the test. The test began at the time the participant's hand entered the water. The participant had been instructed to inform the observer immediately when they first felt pain, and the time difference between the beginning of the test and the first report of pain was recorded as that participant's "pain threshold" [15]. They were instructed to voluntarily withdraw their hand at the point at which the pain became "unbearable" - the time between the beginning of the test and this voluntary withdrawal was recorded as that participant's "pain tolerance" [15].

The cold pressor test was carried out before the participant completed psychological questionnaires. This was in order to prevent the observer from subconsciously influencing the subject's performance based on their "in pain" versus "not in pain" status, or on their results from the psychological measures. 
Participants were aware that a test limit existed for their safety. They were blinded to the actual limit. For safety reasons, the test was terminated after $4 \mathrm{~min}$ (240 s) if the participant had not already removed their hand. The test limit of $4 \mathrm{~min}$ was chosen to limit the risk of tissue injury, as supported by the literature [21-23].

Surgical and anaesthetic records were obtained with participants' informed consent from the Clinical Records Department of Christchurch Public Hospital. All relevant factors that could be located were recorded. These included: indication for surgery; surgical approach; surgical duration; postoperative complications; anaesthetic technique (general anaesthesia or not); and medications (intra-operative, postoperative, and at discharge).

For the sake of analysis, because of the small total sample size $(n=54)$, those in pain at 6 weeks postoperatively were combined with those in pain at 3 months to give one "prolonged pain" group $(n=11)$ [8]. There were eight in pain at 6 weeks. There were four in pain at 3 months, with one patient in pain at both assessments. There were three patients who experienced pain at 3 months whose pain was sub-threshold at 6 weeks.

Data were entered into Microsoft Excel spreadsheets, then into OpenEpi (Dean et al., Open Source Epidemiologic Statistics for Public Health) for quantitative analysis. The $95 \%$ confidence intervals $(95 \% \mathrm{CI})$ were calculated using the score (Wilson) method. $T$ tests (dependent and independent), Chi squared tests, and others were performed as required. Receiver operating characteristic (ROC) curves [24] were calculated using Proc Logistic in SAS/ STAT $^{\circledR} 12.1$ User's Guide. Cary, NC, USA: SAS Institute Inc, 2012. Wald confidence intervals were used for the area under the ROC curves, and comparison of these areas took account of both areas being calculated on the same group of patients.

\section{RESULTS}

\section{Cold Pressor Test Results and Baseline Pain}

Table 1 shows the cold pressure test (CPT) results by baseline pain status.

The truncation of the cold pressor test at 4 min had little impact on pain threshold times as only $2 / 51$ patients reached this limit and none of them developed prolonged pain. For tolerance $12 / 51$ patients were stopped after this time, two of whom developed prolonged pain.

Threshold and tolerance times did not differ significantly between those in pain at baseline and those not in pain at that time (see Table 1).

\section{ROC Curves for Prolonged Pain}

Of the 54 patients, three had no follow-up so could not be assessed for prolonged pain. There were 11 patients with prolonged pain and 40 without such pain. Receiver operating characteristic (ROC) curves were used to show if the cold pressor test measures at baseline were able to distinguish patients who would develop prolonged pain from those who did not. ROC curves plot sensitivity against the false alarm rate (1-specificity) for all possible cut points on a test. If sensitivity and (1-specificity) are equal, then a test is of no use. This would be shown on an ROC plot as a straight line joining $(0,0)$ and $(1,1)$, and the area under the curve (AUC) would be 0.5 .

Figure 1 shows the overlaid ROC curves for prolonged pain with time to pain threshold or time to pain tolerance as potential indicators of prolonged pain. Shorter times are used to indicate prolonged pain. For example, for the 
Table 1 Cold pressor test (CPT) results by baseline pain status

\begin{tabular}{lccc}
\hline & $\begin{array}{l}\text { Not in pain at baseline } \\
(\boldsymbol{n}=\mathbf{2 6})(\mathbf{S D})\end{array}$ & $\begin{array}{l}\text { In pain at baseline } \\
(\boldsymbol{n}=\mathbf{2 8})(\mathbf{S D})\end{array}$ & $\begin{array}{l}\text { Mean difference (95 } \\
\text { \% CI) }\end{array}$ \\
\hline Threshold & $57.0(59.4)$ & $50.8(47.1)$ & $6.2(-23.0,33.4)$ \\
Tolerance & $119.6(73.4)$ & $112.43(88.0)$ & $7.15(-37.3,51.6)$ \\
$\begin{array}{l}\text { Number who reached the tolerance } \\
\text { threshold }(240 \mathrm{~s})\end{array}$ & $5(19.2 \%)$ & $7(25 \%)$ & $\begin{array}{c}\text { Total: } 12 / 54(22.2 \\
\%)\end{array}$ \\
\hline
\end{tabular}

CPT cold pressor test, $S D$ standard deviation

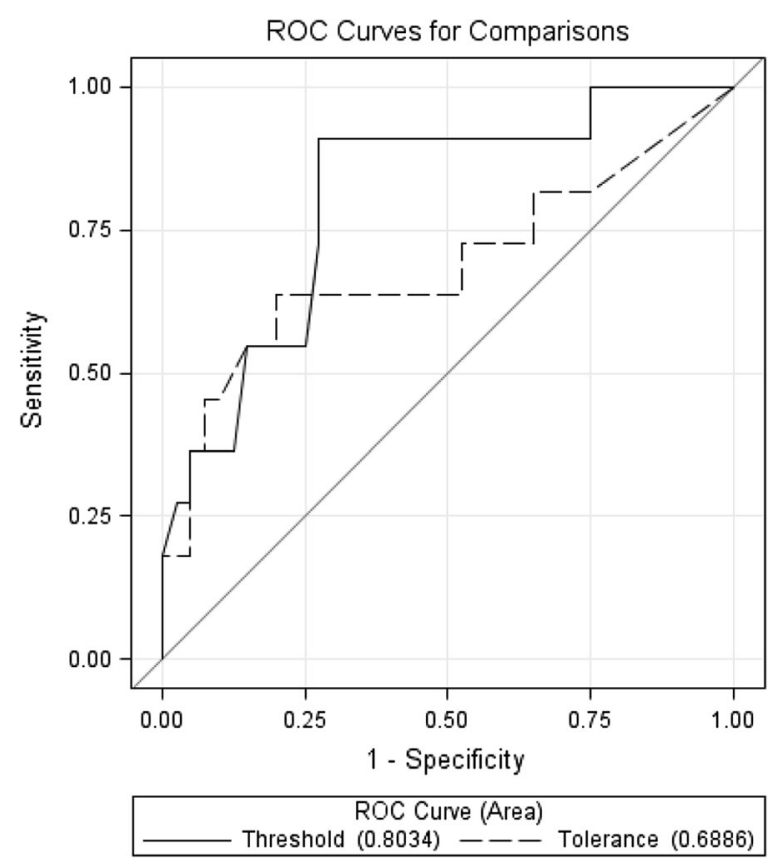

Fig. 1 ROC curves for prolonged pain with pain threshold time or pain threshold tolerance as indicators

pain threshold ROC with a cut point of $<27$ / $\geq 27 \mathrm{~s}$, sensitivity is $8 / 11=0.73$ with (1-specificity) of $11 / 40=0.28$, whereas with a cut point of $<40 / \geq 40 \mathrm{~s}$ sensitivity is $10 / 11=0.91$ but (1-specificity) has risen to 20/40, namely 0.50 . The AUC for time to pain threshold is 0.80 (95\% CI $0.66,0.95)$ which is significantly above 0.50 . The AUC for pain tolerance in lower at 0.69 (95\% CI 0.47, 0.90) so is higher than 0.5 , but not significantly so. The truncation at $240 \mathrm{~s}$ may have somewhat reduced the AUC for tolerance. Because of the relatively small sample size the confidence intervals are wide and these two ROC values are not significantly different $(p=0.19)$.

\section{Surgical Data}

Participants were grouped by surgical groups according to their primary indication for surgery or underlying pathology. Surgical groups were as follows: excision of endometriosis; hysterectomy; hysterectomy; and salpingectomy and/or oophorectomy; salpingectomy and/or oophorectomy; pelvic floor/vaginal wall repair; and other surgeries (in eight patients or $14.4 \%$ ). There were 36 patients $(66.7 \%)$ that underwent laparoscopic surgeries, seven patients $(13.0 \%)$ that underwent laparotomies, and 11 patients (20.4 $\%$ that underwent vaginal surgeries. Laparotomies appeared to be most strongly associated with adverse pain outcomes [8]. It was found that $27.3 \%$ of those who developed prolonged pain, compared with $9.1 \%$ of those who did not develop prolonged pain, had undergone laparotomies. However, this finding was non-significant $(p=0.075)$ [8].

\section{Anaesthetic and Analgesic Data}

All 54 surgeries were performed under general anaesthesia (GA). 


\section{DISCUSSION}

\section{Quantitative Sensory Testing}

In the ongoing effort to predict patient populations at risk for prolonged postoperative pain the use of quantitative sensory testing (QST) has shown promise [25]. These tests aim to measure patients' responses to, and ability to tolerate, pain by applying one of a range of mild to-moderate mechanical, electrical, or thermal stimuli. The results of QSTs may be used to define the territory and pathways of pain mechanisms (i.e. sensory mapping), and have also been touted as ways to identify sensory phenotypes of pain mechanisms [26]. Despite their popularity (likely due to their patient palatability and practitioner convenience), the various forms of preoperative psychometric evaluation are not as efficient as QST in predicting postoperative pain [27]. Key examples of the use and effectiveness of such preoperative stimuli are discussed, alongside the findings from the present study.

\section{Mechanical (or Pressure)}

The intensity of acute postoperative pain in patients undergoing hysterectomy was associated with results from three preoperative mechanical pain stimuli. These were, namely, preoperative brush-evoked allodynia (pain sensation evoked by non-noxious stimuli), pinprick hyperalgesia (increased sensitivity to painful stimuli), and vaginal pressure pain threshold $(p=0.04,<0.01, \quad$ and $<0.01$, respectively). In addition, preoperative brush-evoked allodynia was also associated with pelvic pain 4 months after surgery $(\mathrm{p}<0.01)$ [28].

\section{Electrical}

Regarding the intensity of acute postoperative pain, pain threshold testing with electrical stimuli demonstrates greater predictive power than either mechanical or thermal stimuli $[27,29]$. The pain thresholds measured by preoperative electrical pain stimuli were significantly correlated with postoperative opioid consumption and pain control following percutaneous nephrolithotomy [29]. Preoperative measurement of patients' electrical pain thresholds may also be useful as a screening tool to identify patients at high risk of postoperative pain [29]. However, by nature electrical stimuli may be a less palatable option for many patients than mechanical or thermal stimuli. This may limit its usefulness in the context of routine screening, particularly given that it is plausible that those most at risk would also be more likely to refuse such a test.

\section{Thermal Stimuli (Heat Pain)}

A 2011 previous systematic review [31] conducted a literature search of MEDLINE, EMBASE, and meeting abstracts found 15 studies ( $n=948$ patients) with univariate and/ or multivariate analysis on the topic. The authors divided the painful stimuli applied into three main "types", namely, thermal, pressure (or mechanical), and electrical pain stimuli. The review concluded that the intensity of supra-threshold heat pain (i.e., a patient's perception of pain when receiving a stimulus inducing pain beyond their threshold) correlated most consistently with postoperative pain [30]. In children, a meta-analysis of heat pain found that boys had significantly higher tolerance than girls 
overall. In studies in which the mean age of participants was 12 years or younger, boys were found to have significantly higher heat pain thresholds than girls. These findings suggest that developmental stage may be relevant for understanding sex differences in pain [31], although whether the predictive value of various pain measures regarding prolonged postoperative pain holds true in children is presently unknown.

\section{Cold Pressor Test}

In the present study, at baseline "those in pain" scored slightly lower (albeit not significantly so) than "those not in pain" on all measures. Preoperative cold pressor scores for time to pain threshold and tolerance appeared to predict the likelihood of experiencing prolonged pain (at 6 weeks and/or 3 months, although the AUC was significant only for time to pain threshold.

Ours is not the first pain-prediction study to make use of the cold pressor test. A study of 23 patients (16 women, seven men) scheduled for lower third molar extraction [32] found "preoperative unpleasantness related to the nociceptive stimulus" to be the best predictor of maximum postoperative pain intensity (adjusted $R^{2}=0.39, \quad p=0.001$ ) [32]. A systematic review of the use of the cold pressor task in paediatric pain research has been published [15]. Thirty-six papers, involving 2242 children (aged 3-18 years) from both healthy and clinical samples, met the review's inclusion criteria. Of particular interest, the review found inconsistencies in implementation and reporting in several aspects of CPT methodology with significant potential to impact observed pain outcomes. These included the water temperature used, the use of informed versus uninformed time-ceilings, as well as the presence of observers during the CPT [15]. In the present study, water temperature was constant for all participants $\left(4^{\circ} \mathrm{C}\right)$, measured by thermometer. Participants were informed that a time limit existed for their safety, but were blinded to its value to avoid "targeting". Observers other than the researcher were rare, and were instructed to remain silent and out of the participant's sight during the test.

Interestingly, one study reported that the majority of opioid-dependent male patients on methadone treatment were found to be "pain-sensitive" with the CPT [33]. Poor sleep quality has been associated with cold pressor pain-sensitivity as well [33].

If a way can be found to neutralise the effect of the time limit, a preoperative cold pressor test could prove a useful clinical tool in predicting prolonged pain experience following surgery. In the interest of accuracy at the level of the individual, its predictive powers would need to be combined with other known predictive factors and the patient's clinical picture. Perceived control over anxiety-inducing stimuli such as the cold pressor test increases pain tolerance [16]. Given that the participant had total control over the cold pressor test, it is likely that pain tolerance in this test was as high as could be for patients, except for those who reached the 4 min limit. Perceived control has been found elsewhere to have no influence on pain threshold or intensity [16]. In our study, as this perceived control over the cold pressor test existed in the same manner for all participants, it is unlikely that this produced any bias or confounders in the results. It is the belief of the authors that the cold pressor test would likely be more palatable to patients than alternatives such as electrical impulses. Ice-water carries few strongly negative connotations in the popular mind, while electric shocks, burns, sharp 
objects, and the like may provoke unnecessary anxiety during an already-stressful time (preoperatively).

\section{Perioperative Psychological Risk Factors}

Preoperative psychological risk factors include high preoperative anxiety, surgical fear, depression, and pain catastrophizing [20]. Postoperative psychological risk factors include pain hypervigilance, emotional numbing, and low expectation of return to work [20]. Social and environmental factors include: lower education, lower socioeconomic status, low self-rated health, stressful life events, solicitous responding from significant others, and lack of social support [20].

Psychological factors influencing Gynaecological surgeries are similar to those in other surgical areas. For example, depression, anxiety, and IL- 6 have been found to exacerbate pain during the recovery period following surgery for a gynaecologic malignancy [34]. Another example is that it has been found that pre-existing psychological distress needs to be addressed in young cancer patients undergoing female fertility preservation [35].

A recent Cochrane Systematic Review suggested that psychological preparation may be beneficial for the outcomes for postoperative pain, behavioural recovery, negative affect and length of stay [36]. The psychometric questionnaires used frequently detected differences between those "in pain" and those "not in pain" at given observation points. These were from this same prospective cohort that we have previously published in this journal [8]. We found in the "in pain" group, baseline depression, anxiety, stress, and total DASS-21 scores were all higher than in the "not in pain" group [8]. The association between pain and negative affect was reinforced. No direction of causality could be directly recognised from this data. At all three follow-up points, depression, anxiety, and stress sub-scale scores were associated with the pain state [8].

We found the Brief Illness Perception Questionnaire (BIPQ) to be a potential "screening"-type tool for the risk of developing "prolonged pain." When the BIPQ scores from the baseline assessment were compared between the "prolonged pain" group and the rest of the cohort, BIPQ scores were significantly higher in the "prolonged pain" group (mean difference 7.4, $p=0.049$ ) [8]. Although this difference was not large enough to precisely predict each individual with the potential to develop "prolonged pain", it could be used as a guide for future clinical practice alongside other predictive measures [8].

\section{Types of Surgery}

Type of surgery and surgical approach were not significantly associated with the development of prolonged pain, although results of previous research suggests these associations were likely present but undetectable due to sample size limitations.

\section{Surgical, Anaesthetic, and Analgesic Data}

No surgical, anaesthetic, or analgesic variable was found to be significantly predictive of prolonged postoperative pain. However, this is most likely the result of the number of possible options (large) and the sample size in the study (relatively small), as previous research indicates significant effects for several of these variables [8]. For example, surgery by laparotomy, which came closest to significance in this study $(p=0.075)$ has a well-demonstrated association with prolonged pain [8]. Therefore, such effects most likely existed within the 
present sample, but were too minor to be detected.

\section{Limitations of the Study}

Pain that persists after healing is expected to have taken place, or which exists in the absence of tissue damage, is termed chronic pain [11]. Less than the optimal numbers of participants were enrolled due to time constraints. Most of the patients $(66.7 \%)$ underwent laparoscopic surgeries that are of a minor surgical nature, and would not normally have a high incidence of chronic postoperative pain. This meant that study power was somewhat compromised [10]. Looking at gender, women may have different psychological issues contributing to persisting pain apart from the surgery itself. Future studies should look at gender neutral surgeries. Unlike our study, most studies use a circulating water bath to prevent stagnant water becoming warm around the immersed hand and affecting the result. Chronic pain is usually arbitrarily defined as pain lasting $>3$ or $>6$ months [11]. Time constraints forced the time point at which pain was defined as "chronic" to be 3 months had little effect. On the other hand, if chronic pain is defined as "pain persisting beyond the accepted period of healing", 3 months is well beyond this point.

Only five $(9.3 \%)$ of the fifty four patients dropped out and only three had no follow-up at all. This meant that loss to follow-up was not a major issue. Telephone contact involved multiple attempts.

\section{CONCLUSION}

Although it is recognized that medical and surgical procedures may lead to persistent postoperative pain, predicting which patients are at risk for developing chronic pain presents an ongoing challenge [1]. However, "pain threshold" as measured by this test was shown to predict outcomes of persistent or prolonged pain. Pain tolerance followed the same trend, but was not statistically significant.

To identify the contribution of relevant determinants and modulators of postoperative pain, large prospective-controlled clinical studies are required [37]. New light is shed on the preoperative predictive value of preoperative cold pressor test with pain threshold significantly predictive of prolonged postoperative pain. Further study is needed to develop an arsenal of tools that clinicians can use to identify those at high risk of adverse pain outcomes. It is hoped that in future, timely identification of at-risk individuals may allow for the development and rational use of preventative interventions.

\section{ACKNOWLEDGMENTS}

No funding or sponsorship was received for this study or publication of this article. All named authors meet the International Committee of Medical Journal Editors (ICMJE) criteria for authorship for this manuscript, take responsibility for the integrity of the work as a whole, and have given final approval for the version to be published.

Disclosures. Cameron MacLachlan, Edward A. Shipton, and J. Elisabeth Wells have nothing to disclose.

Compliance with Ethics Guidelines. All procedures followed were in accordance with the ethical standards of the responsible committee on human experimentation (institutional and national) and with the 
Helsinki Declaration of 1964, as revised in 2013. Informed consent was obtained from all patients for being included in the study.

Open Access. This article is distributed under the terms of the Creative Commons Attribution-NonCommercial 4.0 International License (http://creativecommons.org/licenses/ by-nc/4.0/), which permits any noncommercial use, distribution, and reproduction in any medium, provided you give appropriate credit to the original author(s) and the source, provide a link to the Creative Commons license, and indicate if changes were made.

\section{REFERENCES}

1. Kehlet H, Jensen TS, Woolf CJ. Persistent postsurgical pain: risk factors and prevention. Lancet. 2006;367(9522):1618-25.

2. Neil MJ, Macrae WA. Post surgical pain-the transition from acute to chronic pain. Rev Pain. 2009;3(2):6-9.

3. Pogatzki-Zahn ASE. Prädiktoren für chronische Schmerzen nach Operationen. Was wissen wir wirklich? (Predictors of chronic pain following surgery. What do we know?). Der Schmertz. 2010;24:517-33.

4. Shipton EA. Recognition of the vocational practice of the scope of pain medicine in New Zealand. NZ Med J. 2013;126:1368.

5. Smith BH, Torrance N, Bennett MI, Lee AJ. Health and quality of life associated with chronic pain of predominantly neuropathic origin in the community. Clin J Pain. 2007;23(2):143-9.

6. Granot M. Can we predict persistent postoperative pain by testing preoperative experimental pain? Curr Opin Anaesthesiol. 2009;22(3):425-30.

7. Zheng B, Ren M, Lin F, Yao L. Prediction of pain in orthodontic patients based on preoperative pain assessment. Patient Prefer Adher. 2016;10:251-6.

8. MacLachlan C, Shipton EA, Wells JE. Perioperative pain correlates and prolonged post-operative pain predictors: demographics and psychometric questionnaires. Pain Ther. 2015;4(1):119-33.
9. Strulov L, Zimmer EZ, Granot M, Tamir A, Jakobi P, Lowenstein L. Pain catastrophizing, response to experimental heat stimuli, and postcesarean section pain. J Pain. 2007;8(3):273-9.

10. Pan PH, Coghill R, Houle TT, Seid MH, Lindel WM, Parker RL, et al. Multifactorial preoperative predictors for postcesarean section pain and analgesic requirement. Anesthesiology. 2006;104(3):417-25.

11. Hylands-White N, Duarte RV, Raphael JH. An overview of treatment approaches for chronic pain management. Rheumatol Int. 2016. (In press).

12. Mitchell LA, MacDonald RA, Brodie EE. Temperature and the cold pressor test. J Pain. 2004;5(4):233-7.

13. Werner MU, Mjöbo HN, Nielsen PR, Rudin A. Prediction of postoperative pain: a systematic review of predictive experimental pain studies. Anesthesiology. 2010;112(6):1494-502.

14. Lunn TH, Gaarn-Larsen L, Kehlet H. Prediction of postoperative pain by preoperative pain response to heat stimulation in total knee arthroplasty. Pain. 2013;154(9):1878-85.

15. Birnie KA, Petter M, Boerner KE, Noel M, Chambers CT. Contemporary use of the cold pressor task in pediatric pain research: a systematic review of methods. J Pain. 2012;13(9):817-26.

16. Feldner MT, Hekmat H. Perceived control over anxiety-related events as a predictor of pain behaviors in a cold pressor task. J Behav Ther Exp Psychiatry. 2001;32(4):191-202.

17. von Baeyer CL, Piira T, Chambers CT. Guidelines for the cold pressor task as an experimental pain stimulus for use with children. J Pain. 2005;6(4):218-27.

18. Katz J, Seltzer Z. Transition from acute to chronic postsurgical pain: risk factors and protective factors. Expert Rev Neurother. 2009;9(5):723-44.

19. Peters ML, Sommer M, de Rijke JM, Kessels F, Heineman E, Patijn J, et al. Somatic and psychologic predictors of long-term unfavorable outcome after surgical intervention. Ann Surg. 2007;245(3):487-94.

20. Shipton EA. The transition of acute postoperative pain to chronic pain: part 1-risk factors for the development of postoperative acute persistent pain. Trends Anaesth Crit Care. 2014;4:67-70.

21. Moro PJ, Flavian A, Jacquier A, Kober F, Quilici J, Gaborit B, et al. Gender differences in response to cold pressor test assessed with velocity-encoded 
cardiovascular magnetic resonance of the coronary sinus. J Cardiovasc Magn Reson. 2011;13:54.

22. Mourot L, Bouhaddi M, Regnard J. Effects of the cold pressor test on cardiac autonomic control in normal subjects. Physiol Res. 2009;58(1):83-91.

23. Silverthorn DU, Michael J. Cold stress and the cold pressor test. Adv Physiol Educ. 2013;37(1):93-6.

24. Hanley JA, McNeil BJ. The meaning and use of the area under a receiver operating characteristic (ROC) curve. Radiology. 1982;143(1):29-36.

25. Arendt-Nielsen L, Yarnitsky D. Experimental and clinical applications of quantitative sensory testing applied to skin, muscles and viscera. J Pain. 2009;10:556-72.

26. Uddin Z, MacDermid Quantitative sensory testing in musculoskeletal pain.

JC. chronic 2016;17(9):1694-703.

27. Pozek JP, Beausang D, Baratta JL, Viscusi ER. The acute to chronic pain transition: can chronic pain be prevented? Med Clin North Am. 2016;100(1):17-30.

28. Brandsborg B, Dueholm M, Kehlet H, Jensen TS, Nikolajsen L. Mechanosensitivity before and after hysterectomy: a prospective study on the prediction of acute and chronic postoperative pain. Br J Anaesth. 2011;107(6):940-7.

29. Pedersen KV, Olesen AE, Osther PJ, Arendt-Nielsen L, Drewes AM. Prediction of postoperative pain after percutaneous nephrolithotomy: can preoperative experimental pain assessment identify patients at risk? Urolithiasis. 2013;41(2):169-77.

30. Abrishami A, Chan J, Chung F, Wong J. Preoperative pain sensitivity and its correlation with postoperative pain and analgesic consumption: a qualitative systematic review. Anesthesiology. 2011;114(2):445-57.

31. Boerner KE, Birnie KA, Caes L, Schinkel M, Chambers CT. Sex differences in experimental pain among healthy children: a systematic review and meta-analysis. Pain. 2014;155(5):983-93.

32. Mobilio N, Gremigni P, Pramstraller M, Vecchiatini $R$, Calura G, Catapano S. Explaining pain after lower third molar extraction by preoperative pain assessment. J Oral Maxillofac Surg. 2011;69(11):2731-8.

33. Zahari Z, Lee CS, Tan SC, Mohamad N, Lee YY, Ismail R. Relationship between cold pressor pain-sensitivity and sleep quality in opioid-dependent males on methadone treatment. Peer J. 2015;3:e839.

34. Honerlaw KR, Rumble ME, Rose SL, Coe CL, Costanzo ES. Biopsychosocial predictors of pain among women recovering from surgery for endometrial cancer. Gynecol Oncol. 2016;140(2):301-6.

35. Lawson AK, Klock SC, Pavone ME, Hirshfeld-Cytron J, Smith KN, Kazer RR. Psychological counseling of female fertility preservation patients. J Psychosoc Oncol. 2015;33(4):333-53.

36. Powell R, Scott NW, Manyande A, Bruce J, Vögele C, Byrne-Davis LM, Unsworth M, Osmer C, Johnston M. Psychological preparation and postoperative outcomes for adults undergoing surgery under general anaesthesia. Cochrane Database Syst Rev. 2016;(5):CD008646.

37. Shipton EA. The transition of acute postoperative pain to chronic pain: part 2-limiting the transition. Trends Anaesth Crit Care. 2014;4:71-5. 